\title{
Long Term Follow-Up Results of a Fractional Radio Frequency Ablative Treatment of Acne Vulgaris and Related Acne Scars
}

\author{
Judith Hellman \\ Mt. Sinai Hospital, New York, USA \\ Email: jhderm@gmail.com
}

Received 5 April 2016; accepted 30 May 2016; published 2 June 2016

Copyright (C) 2016 by author and Scientific Research Publishing Inc.

This work is licensed under the Creative Commons Attribution International License (CC BY). http://creativecommons.org/licenses/by/4.0/

(c) (i) Open Access

\section{Abstract}

Introduction: Acne vulgaris and acne scarring are prevalent conditions that can have a negative effect on a patient's quality of life. Fractional radiofrequency technologies have been shown to be clinically safe and effective in managing acne scars through dermal remodeling without causing direct damage to the epidermis. In a recently published study, we presented our clinical and histological results in the treatment of patients with active acne and acne related scarring using a Fractional RF (FRF) device. In the current article we demonstrate long term follow-up results, up to two years post last fractional treatment. Methods: Four out of the eight patients who completed a four treatment regimen were invited for long term follow-up visit to document treatment results. In some cases, touch-up treatments were conducted to optimize clinical results. Results: Patients demonstrated significant improvement of acne lesions, acne scarring, pores and skin texture. Long term photos demonstrated that clinical improvement progressed with time. Conclusion: The current study further supports the previous findings that FRF is a safe and effective treatment modality for active acne and acne related scars. Treatment protocol can be customized according to patient needs and clinical results last for long term.

\section{Keywords}

Acne Vulgaris, Fractional Radiofrequency, Acne Scars, Long Term Follow-Up

\section{Introduction}

Facial acne vulgaris and acne scarring are widely prevalent conditions that can have a negative effect on a patient's quality of life. A lot of research has been conducted in an attempt to treat active acne lesions and to mi- 
nimize the appearance of resulting acne scars. Various therapeutic measures such as chemical peeling, dermabrasion, fillers and punch techniques have been performed to improve acne scarring but with sub-optimal outcomes. The potential of ablative lasers such as Er:YAG or $\mathrm{CO}_{2}$ lasers in producing significant improvement was promising; however, these technologies were found to be associated with long recovery times and side effects such as post-inflammatory hyperpigmentation and scarring [1]. More recently, newer methods were developed to minimize adverse effects by treatment in a fractional manner, while leaving healthy areas to promote and improve healing process. Ablative fractional resurfacing using the $\mathrm{CO}_{2}$ fractional laser system has demonstrated significant beneficial effects on atrophic acne scars and minor side effects comparing to non-fractional $\mathrm{CO}_{2}$ laser [2].

Newer techniques such as delivering fractional radiofrequency energy through miniature needles have been shown to be clinically efficient in managing acne scars through dermal remodeling, neoelastosis, and neocollagenesis without causing direct damage to the epidermis [3]-[6]. FRF delivered electrical energy to create zones of thermal damage to the dermis in the area treated while sparing adjacent areas which serve as reservoir for wound healing [7].

Published studies on treatments with the various energy-based fractional technologies demonstrate safety and efficacy in the treatment of acne scars. However, most of the studies involve relatively short follow-up periods usually ranging from one month to three months after last treatment, occasionally 6 months [1]-[6]. This relatively short follow-up period may be due to the difficulty in achieving patients' availability, willingness and compliance in returning to the clinic for assessing and documenting results after termination of treatment program.

In a recently published study, we presented our clinical and histological results in the treatment of eight patients with active acne and acne related scarring, using a Fractional RF (FRF) device (Fractora ${ }^{\mathrm{TM}}$, Invasix Ltd. Israel) [8]. Baseline and follow-up photos were presented to visualize clinical results. As customary in similar studies, follow-up pictures were retaken at one- and three-month time-points after the last treatment.

The objective of the current publication is to demonstrate long term follow-up results, up to two years post last fractional treatment, of four out of the eight patients which participated in the previous study.

\section{Methods}

Patients were young adults with history of scarring acne with or without active acne lesions. As specified in the Methods section of the previously published article [8], patients were treated with an array of 24 RF conducting needles. Each needle was $2500 \mu \mathrm{m}$ long (Fractora, Invasix Ltd., Israel). The hand piece was loaded onto the BodyTite $^{\mathrm{TM}}$ (Invasix, Israel) platform. Some of the tips used in this study feature insulating coating along 2000 $\mu \mathrm{m}$ that provided additional epidermal protection for dark skin. Coated or non-coated tips were used according to patient Fitzpatrick skin type [9]. Patients received initial doses of $20-40 \mathrm{~mJ} / \mathrm{pin}$, and the doses were increased each visit, based on patient tolerance. All treated areas received one or two passes based on the treatment site and patient tolerance. Double and triple stacking of pulses was done as needed.

According to the typical treatment protocol, each patient underwent four treatments, usually four weeks apart. Treatments involved only areas of the face. Frontal and side facial photos were taken immediately prior to the first treatment and at follow up visits at 1 and 3 months after the $4^{\text {th }}$ treatment to qualitatively evaluate and compare acne and scar improvement.

In some cases, a custom tailored protocol was adopted according to individual specific needs. In these cases, patients received additional touch-up treatments outside the original protocol as a result of the desire to further improve the scars and texture after active acne was cured.

Patients who chose to return for further evaluation and consideration of additional treatments, were photographed thus at longer follow-up periods and results could be documented.

\section{Results}

The previously published study included 8 patients who completed 4 treatments, 6 females and 2 males with median age of 20.5 years (range 17 - 41). All patients demonstrated significant improvement of acne lesions and acne scarring already post 4 FRF treatments.

The current publication demonstrates long term follow-up results of 4 out of the above 8 patients. In some cases, additional touch-up treatments were provided according to the clinical status of the individual patient. 
Long term results were collected 1 - 2 years after the last treatment according to the specific schedule customized for each patient.

The photos below demonstrate on-going improvement of results from baseline, short term follow-up (4 - 8 weeks post 4 treatments), and long term follow-up results.

Figure 1 shows long term follow-up results 2 years after the original protocol of 4 treatments. A coated 24 pins tip was used to protect the epidermis of this Hispanic patient with Fitzpatrick skin type IV.

The other 3 patients received additional 2 - 3 touch-up treatments on top of the original 4 treatments protocol, according to specific needs for further skin texture improvement.

Figure 2 and Figure 3 demonstrate long term follow up results of 2 patients who received a total of 6 treatments each, for further scars and pores improvement. In both cases coated 24 pins tip was used. One year (12 and 13 months) photos post $6^{\text {th }}$ treatment were collected to demonstrate further improvement compared to photos of 1 month post 4 treatment.

Figure 4 demonstrates results of a patient who underwent a total of 7 treatments with non-coated 24 pins tip due to his light skin type. The 4 initial treatments were conducted once a month according to the original protocol. The additional 3 touch-up treatments were spaced about 5 months apart, according to patient availability. The last follow-up photos were taken 2 years post $4^{\text {th }}$ treatment ( 4 months post $7^{\text {th }}$ treatment).

It should be noted that one more patient who was followed two years after last treatment demonstrated impressive results (not yet photographed at the clinic).
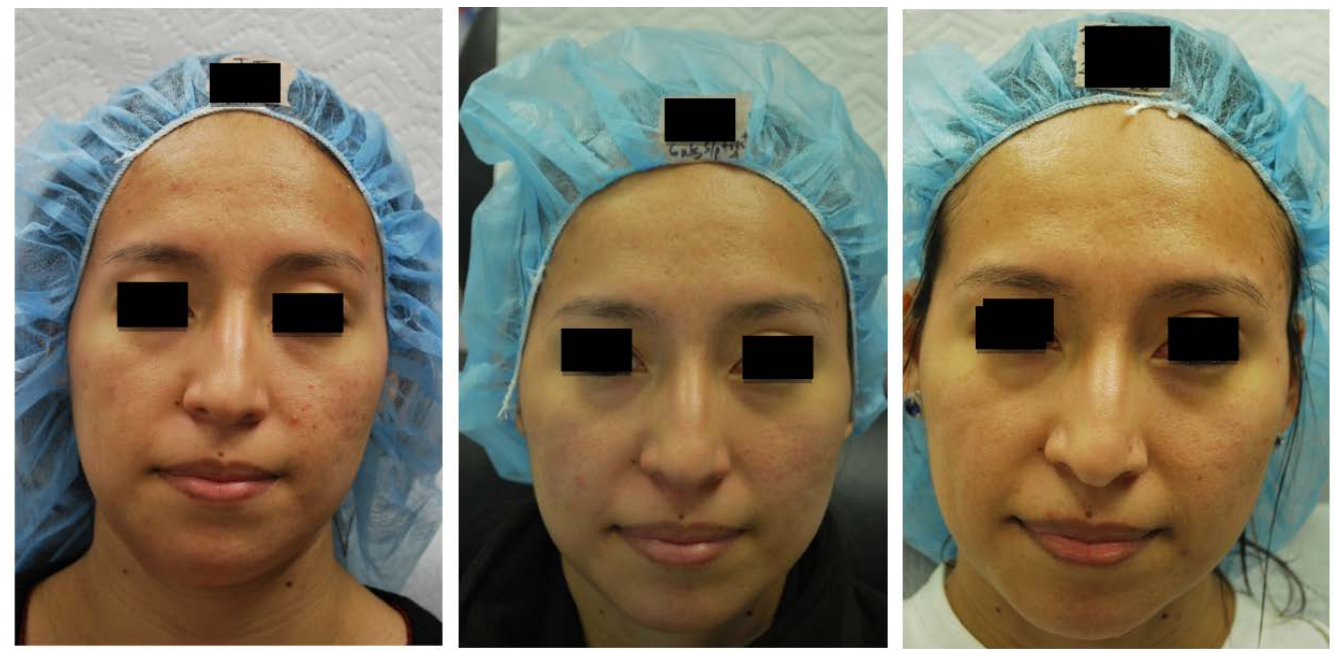

Figure 1. Scarring acne and acne scars before (left), 6 weeks post 4 treatments (middle), and 2 years post 4 treatments (right).
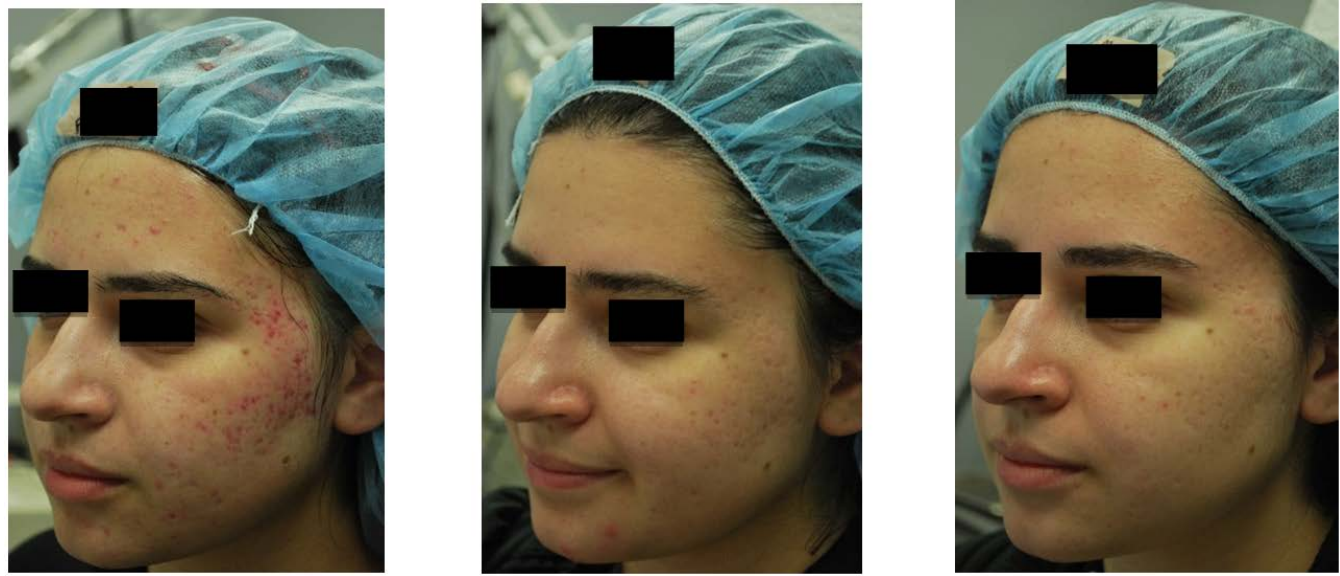

Figure 2. Acne and acne scarring before (left), 1 month post 4 treatments (middle) and 12 months post 6 treatments (right). 

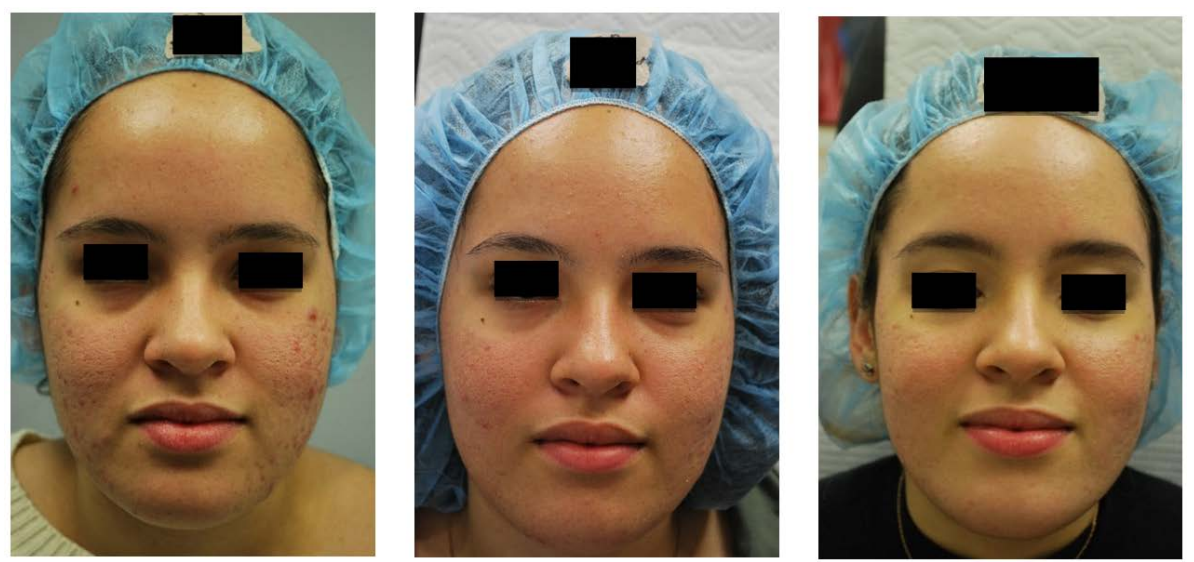

Figure 3. Acne, acne scarring and pores before (left), 1 month post 4 treatments (middle) and 13 months post 6 treatments (right).
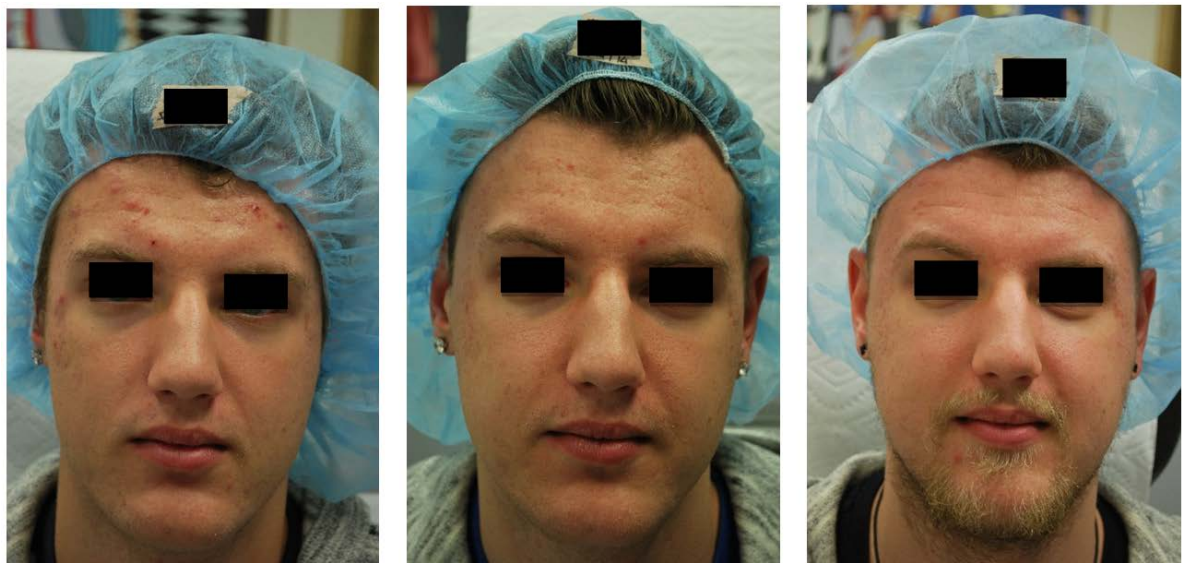

Figure 4. Acne and acne scarring and pores before (left), 2 months post 4 treatments (middle) and 2 years post $4^{\text {th }}$ treatment ( 4 months post $7^{\text {th }}$ Treatment) (right).

\section{Discussion}

The current publication reports long term follow-up of the use of Fractional Radiofrequency (FRF) device for the treatment of active acne lesions, acne scarring, pores and general skin texture improvement. Our previously published article confirmed that 4 treatments with FRF led to significant improvement in the depth of the scars as demonstrated by significant improvement in clinical scoring and by histological evidence [8]. Concomitantly, significant resolution of active acne lesions was observed.

FRF treatment was found to have significant advantages as it is minimally invasive, has very low downtime (most patients required 3 - 4 days of recovery) and safe. We did not encounter any cases of unexpected adverse event, including scarring and pigmentation alteration and therefore the treatment was found to be suitable for dark skin types. The availability of the coated pins tip, optimizes the suitability of the treatment to dark skin patients with a high level of safety.

As noted in the previous article, this is of significant value as adult acne and atrophic scarring may be more prevalent in darker skin types [10].

For the purpose of the study we originally arbitrarily decided on a protocol of four treatments spaced four weeks apart. During the course of the evaluation, it was found that while some patients achieved satisfactory response in 4 treatments, other patients were elected to add more treatments in order to optimize treatment results. Adding a few touch-up treatments was found to be effective in further improving the results and maintain them for a long period. We therefore concluded that the total number of treatments required can be variable and depends on the goals of treatment and the patient's individual response. 
In order to further evaluate and establish the examined FRF treatment, we maintained contact with selected patients to document treatment results in longer periods then what is customary in similar medical aesthetic treatments. Longer follow-ups in this study ranged from 4 months to 2 years. It was found that clinical results of improved skin texture and acne scars sustained for a long period, findings that support the beneficial nature of the FRF treatment.

\section{Conclusion}

The current case-study further supports the previous findings that FRF is a safe and effective treatment modality for both active acne and acne related scars. Both light and dark skin patients benefitted from the FRF treatment regimen. Treatment protocol can be customized according to patient needs and results last for long term. Further larger scale randomized controlled studies are required to fully evaluate this treatment modality for the clinical stubborn phenomenon of acne and acne scars.

\section{References}

[1] Kutlubay, Z. and Gokdemir, G. (2010) Treatment of Atrophic Facial Acne Scars with the Er:YAG Laser: A Turkish Experience. Journal of Cosmetic and Laser Therapy, 12, 65-72. http://dx.doi.org/10.3109/14764171003706141

[2] Manuskiatti, W., Triwongwaranat, D., Varothai, S., et al. (2010) Efficacy and Safety of a Carbon-Dioxide Ablative Fractional Resurfacing Device for Treatment of Atrophic Acne Scars in Asians. Journal of the American Academy of Dermatology, 63, 274-283. http://dx.doi.org/10.1016/j.jaad.2009.08.051

[3] Taub, A.F. and Garretson, C.B. (2011) Treatment of Acne Scars of Skin Types II to V by Sublative Fractional Bipolar Radiofrequency and Bipolar Radiofrequency Combined with Diode Laser. Journal of Clinical and Aesthetic Dermatology, 4, 18-27.

[4] Elman, M. and Harth, Y. (2011) Novel Multi-Source Phase-Controlled Radiofrequency Technology for Non-Ablative and Micro-Ablative Treatment of Wrinkles Lax Skin and Acne Scars. Laser Therapy, 20, 139-144. http://dx.doi.org/10.5978/islsm.20.139

[5] Chandrashekar, B.S., Sriram, R., Mysore, R., et al. (2014) Evaluation of Microneedling Fractional Radiofrequency Device for Treatment of Acne Scars. Journal of Cutaneous and Aesthetic Surgery, 7, 93-97. http://dx.doi.org/10.4103/0974-2077.138328

[6] Zhang, Z., Fei, Y., Chen, X., et al. (2014) Comparison of a Fractional Microplasma Radio Frequency Technology and Carbon Dioxide Fractional Laser for the Treatment of Atrophic Acne Scars: A Randomized Split-Face Clinical Study. Journal of Cutaneous and Aesthetic Surgery, 7, 93-97.

[7] Lolis, M.S. and Goldberg, D.J. (2012) Radiofrequency in Cosmetic Dermatology: A Review. Dermatologic Surgery: Official Publication for American Society for Dermatologic Surgery, 38, 1765-1776.

http://dx.doi.org/10.1111/j.1524-4725.2012.02547.x

[8] Hellman, J. (2015) Retrospective Study of the Use of a Fractional Radio Frequency Ablative Device in the Treatment of Acne Vulgaris and Related Acne Scars. Journal of Cosmetics, Dermatological Sciences and Applications, 5, 311316. http://dx.doi.org/10.4236/jcdsa.2015.54038

[9] Fitzpatrick, T.B. (1988) The Validity and Practicality of Sun-Reactive Skin Types I through VI. Archives of Dermatology, 124, 869-871. http://dx.doi.org/10.1001/archderm.1988.01670060015008

[10] Perkins, A.C., Cheng, C.E., Hillebrand, G.G., Miyamoto, K. and Kimball, A.B. (2011) Comparison of the Epidemiology of Acne Vulgaris among Caucasian, Asian, Continental Indian and African American Women. Journal of the European Academy of Dermatology and Venereology, 25, 1054-1060.

http://dx.doi.org/10.1111/j.1468-3083.2010.03919.x 